\title{
Aberrant chimeric RNA GOLM1-MAK10 encoding a secreted fusion protein as a molecular signature for human esophageal squamous cell carcinoma
}

\author{
Hao Zhang ${ }^{1,2,3}$, Wan Lin ${ }^{2}$, Kalpana Kannan ${ }^{4,5}$, Liming Luo ${ }^{4,5}$, Jing Li ${ }^{6}$, Pei-Wen \\ Chao $^{4,5}$, Yan Wang ${ }^{4,5}$, Yu-Ping Chen ${ }^{7}$, Jiang Gu ${ }^{6}$, Laising Yen ${ }^{4,5}$ \\ ${ }^{1}$ Department of Integrative Oncology, Affiliated Cancer Hospital, Shantou University Medical College, Shantou, Guangdong, \\ China. \\ ${ }^{2}$ Cancer Research Center, Shantou University Medical College, Shantou, Guangdong, China. \\ ${ }^{3}$ Tumor Tissue Bank, Affiliated Cancer Hospital, Shantou University Medical College, Shantou, Guangdong, China. \\ ${ }^{4}$ Department of Pathology \& Immunology, Baylor College of Medicine, Houston, TX, USA. \\ ${ }^{5}$ Department of Molecular and Cellular Biology, Baylor College of Medicine, Houston, TX, USA. \\ ${ }^{6}$ Department of Pathology, Shantou University Medical College, Shantou, Guangdong, China. \\ 7 Department of Thoracic Surgery, Affiliated Cancer Hospital, Shantou University Medical College, Shantou, Guangdong, \\ China. \\ Correspondence to: Hao Zhang, email: haozhang@stu.edu.cn \\ Laising Yen, email: yen@bcm.edu \\ Keywords: Transcriptome, transcription-induced chimeric RNAs, GOLM1-MAK10, secreted fusion protein, cancer biomarker, \\ esophageal cancer. \\ Received: October 7, $2013 \quad$ Accepted: October 30, $2013 \quad$ Published: November 1, 2013
}

This is an open-access article distributed under the terms of the Creative Commons Attribution License, which permits unrestricted use, distribution, and reproduction in any medium, provided the original author and source are credited.

\section{ABSTRACT:}

It is increasingly recognized that chimeric RNAs may exert a novel layer of cellular complexity that contributes to oncogenesis and cancer progression, and could be utilized as molecular biomarkers and therapeutic targets. To date yet no fusion chimeric RNAs have been identified in esophageal cancer, the 6th most frequent cause of cancer death in the world. While analyzing the expression of 32 recurrent cancer chimeric RNAs in esophageal squamous cell carcinoma (ESCC) from patients and cancer cell lines, we identified GOLM1-MAK10, as a highly cancer-enriched chimeric RNA in ESCC. In situ hybridization revealed that the expression of the chimera is largely restricted to cancer cells in patient tumors, and nearly undetectable in nonneoplastic esophageal tissue from normal subjects. The aberrant chimera closely correlated with histologic differentiation and lymph node metastasis. Furthermore, we demonstrate that chimera GOLM1-MAK10 encodes a secreted fusion protein. Mechanistic studies reveal that GOLM1-MAK1O is likely derived from transcription read-through/splicing rather than being generated from a fusion gene. Collectively, these findings provide novel insights into the molecular mechanism involved in ESCC and provide a novel potential target for future therapies. The secreted fusion protein translated from GOLM1-MAK10 could also serve as a unique protein signature detectable by standard non-invasive assays. These observations are critical as there is no clinically useful molecular signature available for detecting this deadly disease or monitoring the treatment response.

\section{INTRODUCTION}

Esophageal squamous cell carcinoma (ESCC) is the third most common malignancy of the digestive tracts $[1,2]$. Patients with ESCC usually present with disease that is locally advanced or already metastasized at the 
time of diagnosis, and are frequently resistant to current chemoradiotherapeutic strategies, resulting in sixth leading cause of cancer death in the world [3]. This is largely due to the limited understanding on molecular mechanisms driving carcinogenesis and progression of this cancer, and the lack of molecular signatures useful for early diagnosis and monitoring therapeutic efficiency.

It is increasingly recognized that chimeric RNAs may exert a novel layer of cellular complexity that contributes to oncogenesis and cancer progression. With the aid of high-throughput RNA sequencing technology and bioinformatics analysis, transcriptome sequencing has uncovered a large number of chimeric RNAs across different human tissues and diseases [4-9]. Studies provide encouraging evidence that signature chimeric RNAs could be identified for the early detection of tumors [6-8]. These chimeric RNAs, which are unrelated to chromosomal rearrangements (fusion genes), are primarily generated by two transcription-induced mechanisms [4, 5] (Figure 1). The first is 'read-through/splicing', where a chimeric RNA begins at the upstream gene and ends at a termination point of a downstream gene, with the region in between removed by splicing. The second is 'trans-splicing', where two separately transcribed RNAs are spliced together to give rise to a single RNA. Both mechanisms result in fused transcripts that possess sequences from two parental genes.

We recently identified 2,369 chimeric RNA candidates by performing high-throughout sequencing analysis of prostate cancer samples, and confirmed 32 highly cancer-enriched chimeric RNAs [6]. The chimeric RNAs are significant in several ways. First, many are highly enriched in tumor and recurrent among patients [6]. Second, some are released in body fluids such as urine at detectable levels [7, 8], and thus could serve as noninvasive biomarkers for PCR-based assay. Third, chimeric RNAs can lead to the translation of fusion proteins [9], hence potential therapeutic targets (Figure 1). An example is the $B C R-A B L$ fusion protein in chronic myeloid leukemia that is now successfully treated by the small molecule drug Gleevec [10]. Despite their importance, chimeric RNAs are largely under-investigated and mostly unknown in esophageal carcinoma.

In this study, we investigated a set of 32 highly recurrent chimeric RNAs recently identified in prostate cancer [6], and characterized their presence in multiple ESCC and non-neoplastic cell lines, in addition to a large cohort of clinical ESCC patient samples. This led to the identification of GOLM1-MAK10 as highly differentially expressed chimeric RNAs in ESCC. Importantly, the expression of the chimera is markedly elevated in cancer cells within patients' tumor and nearly undetectable in esophageal tissue from subjects without esophageal neoplasia. The aberrant chimera closely correlated with tumor differetiation and lymph node metastasis. Moreover, we showed that the chimera GOLM1-MAK10 led to the translation of a fusion protein that was secreted into the cell-culture media. Our results thus suggest that the aberrant chimeric RNA GOLM1-MAK10 is abundant and may represent a novel molecular alteration in ESCC that could have important implications in understanding the mechanism and early detection of ESCC.

\section{Transcription-induced chimeric RNAs}

A Read-through transcription followed by splicing

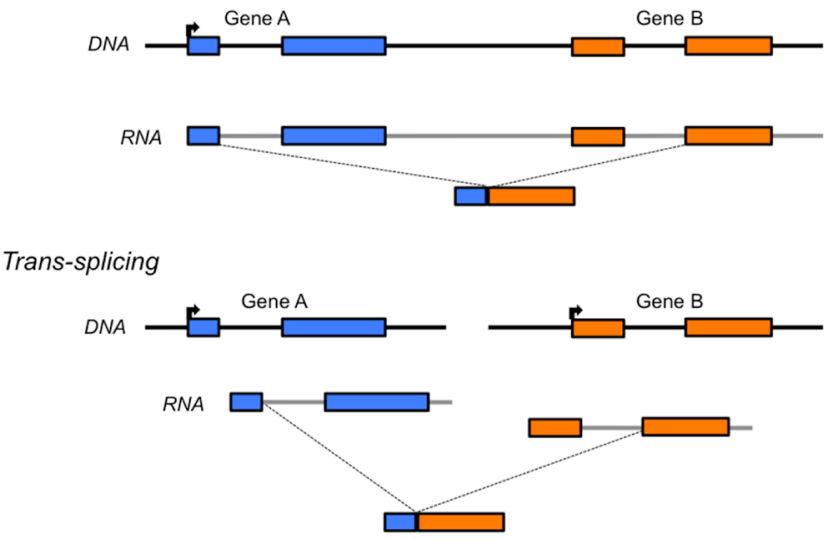

B

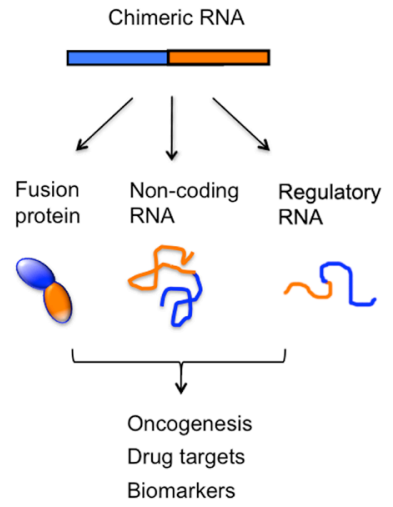

Figure 1: Potential importance of transcription-induced chimeric RNAs. (A) Chimeric RNAs unrelated to chromosomal rearrangements are generated through either 'readthrough/splicing' or 'trans-splicing'. Both mechanisms result in fused transcripts that possess sequences from two parental genes. (B) Such chimeric RNA can give rise to novel fusion proteins, non-coding RNA or regulatory RNA that may be responsible for oncogenesis, and serve as drug targets or useful clinical biomarkers. Primer pairs can be specifically designed to amplify chimeric RNA in patients' tissue without amplifying the parental genes. 


\section{RESULTS}

\section{Identification of GOLM1-MAK10 in ESCC}

A set of 32 cancer-enriched and recurrent chimeric RNAs we recently discovered was chosen for initial screening [6]. To evaluate the expression of chimeric RNA, we performed RT-PCR using primer pairs tailored for each of the 32 chimeras so that each primer of the pair anneals to one parental gene therefore specifically amplifies the chimeric transcript but not the parental gene transcript. The assay was performed on several ESCC cell lines, including EC109, EC9706, HK2, HK3, and TE1, and compared to that of the immortalized normal esophageal epithelial cells such as NE1 and NE3 cells. These chimeric transcripts were confirmed by direct Sanger sequencing of RT-PCR products. In addition, we evaluated eighteen pairs of cancer/matched benign tissue isolated from ESCC patients. RT-PCR showed that out of the 32 chimeric RNAs, 18 displayed varying degrees of expression in cancer vs. matched benign tissue (Figure 2). However, we found conspicuously higher expression of 2 chimeras, namely ASTN2-PAPPA and GOLM1$M A K 10$, in patients' cancer tissue vs. matched benign tissue (Figure 2, 3A), as well as in ESCC cancer cell lines vs. immortalized esophageal cells (Figure 2, 3B). Among

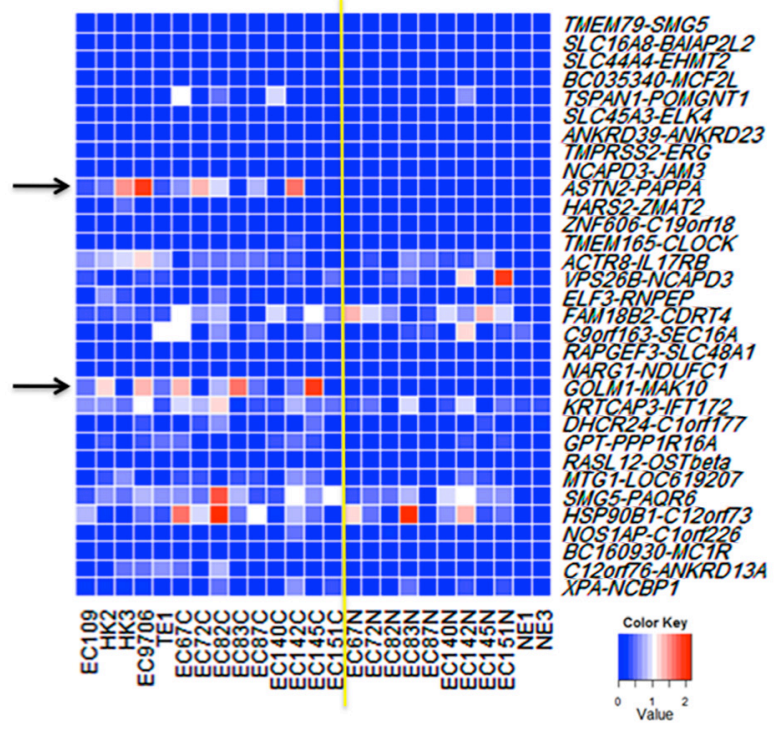

Figure 2: Screening chimeric RNA candidates. Initial screening of 32 chimeric RNAs in ESCC cell lines and patients' tissue was performed by RT-PCR using chimera-specific primers. The expression level was graded based on RT-PCR band intensity. The yellow line demarcates cancer from normal. Left side: ESCC cell lines (EC109, HK2, HK3, EC9706, TE1) and cancer tissues. Right side: matched benign tissues and the immortalized normal esophageal epithelial cells (NE1 and NE3). Most of the chimeric RNAs displayed varying expression levels. ASTN2-PAPPA and GOLM1-MAK10, as marked by arrows, were conspicuously expressed at higher levels in cancer. these two chimeras, we focused on GOLM1-MAK10 in the subsequent studies for the following reasons: 1) the 5' parental gene GOLM1 (also known as GOLPH2 or GP73), a Golgi membrane protein, is predominantly expressed in epithelial cells [14], the cell type that ESCC is derived from; and 2) the chimera GOLM1-MAK10 retains most of the GOLM1 sequence including the signal peptide/ transmembrane domain that are required for secretion (see below and Figure 5). Active secretion is particularly important for cancer detection, considering that the most clinically useful cancer biomarkers to date, such as PSA for prostate cancer and CA125 for ovarian cancer, are secreted proteins.

To determine the cellular origin of GOLM1-MAK10 within patients' tumor, we performed in situ hybridization using antisense probes against the chimeric junctions and segments specific to GOLM1-MAK10. The results revealed strong expression in cancerous esophageal epithelium from ESCC patients with very low staining in the adjacent stromal cells (Figure 3C). This confirmed that the chimera in question is derived primarily from cancer cells within the tumor. The data hence suggest the association of the chimeric GOLM1-MAK10 with ESCC.

\section{Validation of GOLM1-MAK10 in a large cohort of ESCC vs. matched benign tissues and non- neoplastic tissues}

To validate the prevalence of GOLM1-MAK10 in ESCC and determine its clinicopathological significance, a large cohort of paired cancer/matched benign tissues from a total of 115 patients were examined by quantitative RT-PCR analysis. As shown in Figure 4, the chimera GOLM1-MAK10 was expressed at a distinctly higher level in cancer tissues vs. matched benign tissues ( $\mathrm{p}=7.9 \mathrm{e}-07)$. Abberant GOLM1-MAK10 was associated with histologic differentiation and lymph node metastasis $(\mathrm{p}=0.013$ \& 0.023 respectively, Table 1 ), but not statistically signifcant with any other clinicopathological indicators.

To minimize the possible issues of tissue impurity caused by matched benign tissues that may contain multifocal premalignant lesions that precede histological changes [15], 10 esophageal specimens obtained from subjects without esophageal neoplasia were examined. This refined analysis compares cancer tissue from ESCC patients directly to non-neoplastic tissue from normal subjects, thus issues of tissue impurity and premalignant lesions are minimized. The analysis revealed that nonneoplastic tissue from subjects without esophageal neoplasia expressed near undetectable level of chimera, as compared to the remarkably high level in ESCC tissue ( $p=3.7 e-07$, Figure 4$)$. This marked difference in expression level suggests that GOLM1-MAK10 could be a promising indicator to differentiate between ESCC patients and subjects without cancer. 


\section{GOLM1-MAK10 encodes and leads to the translation of a secreted fusion protein}

We next determined if GOLM1-MAK10 translates into a secreted fusion protein. The RNA junction sequences determined by RT-PCR and Sanger sequencing enabled us to analyze the potential protein consequences of GOLM1-MAK10. Our analysis indicated that GOLM1MAK10 could result in the substitution of the last 25 amino acids of GOLM1 by 26 new amino acids and a new 3' UTR from MAK10 (Figure 5A). This would leave the

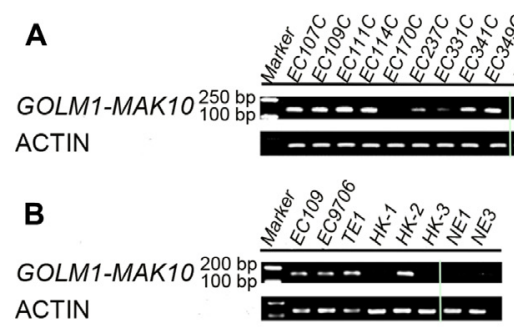

C

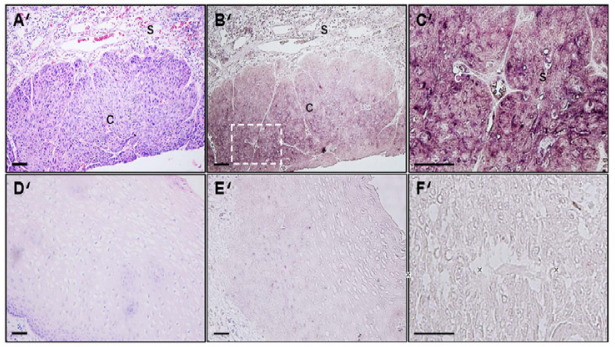

Figure 3: Expression and cellular origin of GOLM1MAK10 in ESCC patient tissues and cancer cell lines. (A) GOLM1-MAK10 was readily detectable by RT-PCR, and differentially expressed in cancer vs. matched benign tissue. The expression of chimeric RNA in 9 patients are shown. Left side: cancer tissues. Right side: matched benign tissues. (B) GOLM1MAK10 was expressed in some ESCC cell lines, including EC109, EC9706, TE1, HK1, HK2, and HK3, but minimally expressed in immortalized esophageal epithelial cells NE1 and NE3. Both (A) and (B) suggest that GOLM1-MAK10 is enriched in cancer cells. (C) Cellular origin of GOLM1-MAK10 RNA in tissue sections of ESCC patient as determined by in situ hybridization. (A'), (B'), (C'), and (F'): cancerous esophageal epithelium. (D') and (E'): non-neoplastic esophageal epithelium. Hematoxylin/eosin staining identifies cancer mass with intense purple color in cancerous esophageal epithelium (A'), which is largely absent in non-neoplastic esophageal epithelium (D'). Hybridization with antisense RNA probe reveals the location of chimeric RNAs with dark maroon color in cancerous esophageal epithelium (B'). Note that the areas positive for chimeric RNAs in B' co-localize with cancer mass in A'. Stromal cells surrounding tumor tissue also showed slight elevated staining but to a lesser extent. In contrast, non-neoplastic esophageal epithelium shows little or no staining with antisense probe (E'). The inset in B' is magnified as C', which reveals the cytoplasmic localization of chimeric RNAs in cancer cells. F' is an adjacent section of C' stained with sense probe as control, which resulted in little signal. s: stromal; c: cancer. Scale bar $=100$ um. signal peptide and transmembrane domain of GOLMI intact [16], hence leading to the secretion of an aberrant fusion protein. To investigate this possibility, we cloned the full length ORF of GOLM1-MAK10 and tagged it with a FLAG sequence (Figure 5B), and transfected it in HEK 293 T cells. Protein analysis confirmed that GOLM1MAK10 is indeed translated into a fusion protein. The size of the fusion protein suggests a dimer formation in a manner similar to that of the parental GOLM1 (Figure 5C and 5D). This is expected, because the coiled-coil domain required for the dimerization of GOLM1 also remains unchanged in GOLM1-MAK10. Importantly, this fusion protein was also found in the cultured media devoid of cellular debris, indicating that it is secreted in a manner similar to its parental protein GOLMI (Figure 5C). Thus, the secreted fusion protein makes GOLM1-MAK10 an attractive candidate as useful biomarker for detecting ESCC.

\section{Mechanisms responsible for generation of GOLM1-MAK10}

To determine if the chimera is the result of genomic DNA rearrangement (fusion gene), we performed longrange PCR on the genomic DNA isolated from patients'

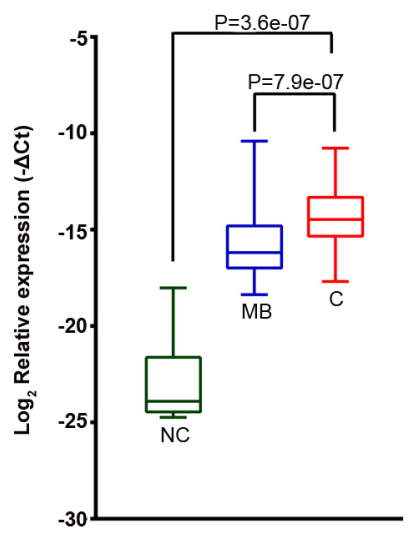

Figure 4: GOLM1-MAK10 was expressed at a significantly higher level in ESCC. The relative expression level of GOLM1-MAK10 chimera as determined by quantitative RT-PCR (normalized to GAPDH). The plot compares the expression in esophageal epithelium of cancer $(\mathrm{C}, \mathrm{n}=78)$, matched benign $(\mathrm{MB}, \mathrm{n}=78)$, and an independent cohort of non-neoplastic esophageal epithelium from normal subjects $(\mathrm{NC}, \mathrm{n}=10)$. GOLM1-MAK10 was expressed at a significantly higher level in cancer vs. matched benign, or vs. non-neoplastic esophageal epithelium. $p$ value of $C$ vs. MB was calculated using paired student $\mathrm{T}$ test, while $\mathrm{C}$ vs. $\mathrm{NC}$ using unpaired student $\mathrm{T}$ test. Y-axis is $(-\Delta \mathrm{Ct})$. Relative fold change in gene expression equals 2 to the power of $(-\Delta \mathrm{Ct})$. Box-plot shows the range of relative expression levels with sample minimum and maximum represented by a short horizontal bar. The box itself represents $50 \%$ of all samples with the lower and upper quartile separated by a line that represents the median. 
A

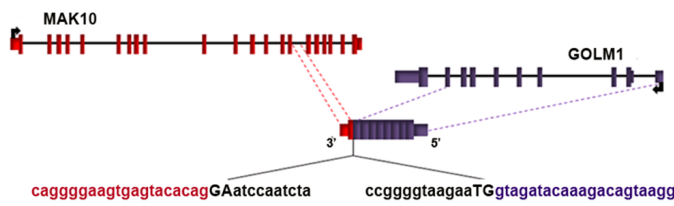

B

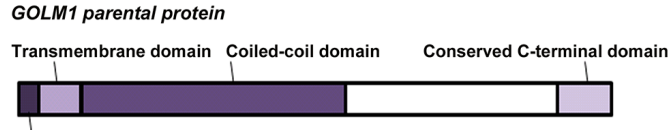

Signal peptide

GOLM1-MAK10 fusion protein Sequence from MAK10 FLAG tag

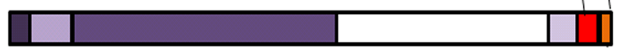

C

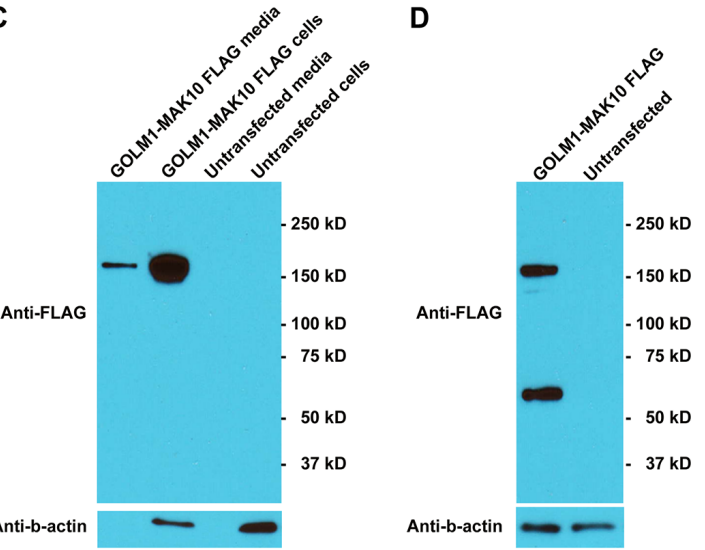

Figure 5: GOLM1-MAK10 leads to the translation of a secreted fusion protein. (A) Schematic of GOLM1-MAK10 chimera. The chimera of GOLM1-MAK10 could result in the substitution of the last 25 amino acids of GOLM1 by 26 new amino acids with the 3' UTR from MAK10. In the pictogram, coding exons are represented by blocks connected by horizontal lines representing introns. The 5'- and 3 '-UTR are represented by shorter blocks. Arrows indicate direction of transcription of parental genes. Dashed lines indicate the boundaries of the sequence contributing to chimeric RNAs. Splice junctions are shown as uppercase letters. The sequences in black are part of the introns removed in the formation of the chimeric RNAs. Note that a discernible 5' splice site and a 3' splice site are present at the RNA junction. (B) Chimera RNA GOLM1-MAK10 is predicted to translate into a fusion protein with the majority of amino acid sequence of GOLM1 retained, and has a near identical size to the GOLM1 parental protein. GOLM1-MAK10 would retain the signal peptide region and the transmembrane domain that are required for secretion. The coiled-coil domain required for the dimerization of GOLM1 also remains unchanged. (C) GOLM1-MAK10 construct with FLAG tag was expressed under the control of the CMV promoter in HEK 293T cells. Fusion protein is efficiently translated and secreted in the media as dimers. For FLAG western, $0.4 \%$ of the total cell extract and $0.4 \%$ of the total media were loaded. For Actin western, equal amounts of extracts were loaded. (D) Iodoacetamide treatment, which reduces the disulfide bonds, partially converted dimers into monomers. The expected size of the monomer is $44 \mathrm{kD}$. However, we observe bands at roughly $60 \mathrm{kD}$ for the monomer, suggesting that they are heavily glycosylated. This is consistent with previous observations of GOLM1. tissue that expressed the corresponding chimeric RNA. The assay yielded indistinguishable product sizes between ESCC tissue, matched benign tissue, and normal human genomic DNA control, indicating that there were no apparent DNA rearrangements in ESCC tissues (Figure 6A). Intriguingly, further sequence analyses indicated that GOLM1-MAK10 RNA contains a discernable 5' splice site and a 3' splice site at the RNA junction (Figure 5A), indicating that the formation of these chimeras is mediated by RNA splicing. Collectively, the observed splice site at

A
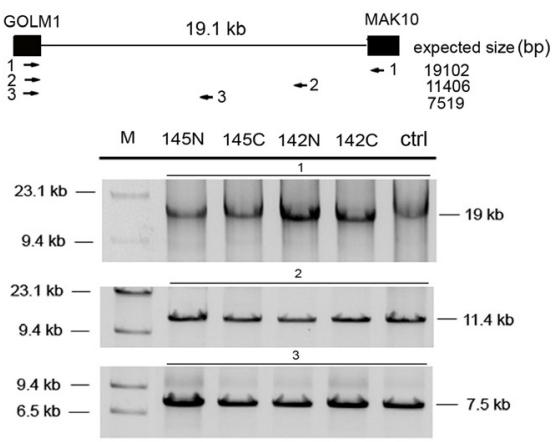

B GOLM1 (P=3.09e-07)
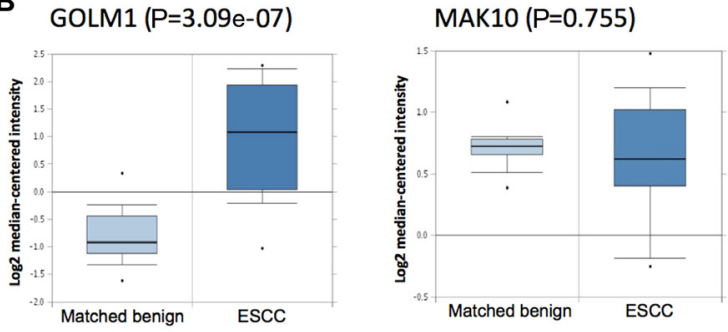

Figure 6: Chimeric GOLM1-MAK10 is generated through transcription-induced mechanisms. (A) Genomic DNA analysis of GOLM1-MAK10 revealed no chromosomal rearrangement. This assay was possible for GOLM1-MAK10 as parental genes are adjacent genes on the same chromosome. Upper panel: diagram of the intergenic region between the RNA junction of GOLM1-MAK10. Black boxes represent the chimeric RNA exons. Three primer pairs were used to scan the $19 \mathrm{~kb}$ intergenic region. The expected product sizes are indicated below the diagram. Lower panel: results of longrange PCR. Results from two ESCC patients (145C and 142C), the matched benign esophageal tissues $(145 \mathrm{~N}$ and142N), and normal human genomic DNA from Promega (ctrl) were shown. Absence of size differences between cancer, benign, and normal control indicates that there were no gross DNA rearrangements. (B) The 5' parental gene GOLM1 is over-expressed in ESCC. The microarray expression analysis was based on the published data by $\mathrm{Hu}$ et al 2010 available from Oncomine (17). The plot compares the expression in esophageal epithelium of matched benign $(n=17)$ vs. esophageal epithelium of cancer $(n=17)$. The 5' parental gene was over-expressed in cancer vs. matched benign $(p$ value $=3.09 e-07)$, but not the 3 ' parental gene $(p$ value $=0.775)$. $Y$-axis represents relative gene expression in $\log 2$ scale. Box-plot shows the range of relative expression levels with sample minimum and maximum represented by a short horizontal bar. The box itself represents $50 \%$ of all samples with the lower and upper quartile separated by a line that represents the median. Dots outside the bar are considered outliers. 
Table 1: Correlation between GOLM1MAK10 level and clinicopathologic variables in patients with ESCC

\begin{tabular}{|l|l|l|l|l|}
\hline Variables & $\begin{array}{l}\text { No. of } \\
\text { patients }\end{array}$ & \multicolumn{2}{l|}{ GOLM1-MAK10 } & \\
\hline & & Low (\%) & High (\%) & $\mathrm{P}^{*}$ \\
\hline Total samples & 78 & $39(50.0)$ & $39(50.0)$ & \\
\hline Sex & & & & \\
\hline Male & 60 & $31(51.7)$ & $29(48.3)$ & 0.591 \\
\hline Female & 18 & $8(44.4)$ & $10(55.6)$ & \\
\hline Age (years) & & & & \\
\hline$\leq 60$ & 39 & $20(51.3)$ & $19(48.7)$ & 0.821 \\
\hline$>60$ & 39 & $19(48.7)$ & $20(51.3)$ & \\
\hline Tumor location & & & & \\
\hline Upper/Middle & 68 & $35(51.5)$ & $33(48.5)$ & 0.498 \\
\hline Lower & 10 & $4(40.0)$ & $6(60.0)$ & \\
\hline Tumor size & & & & \\
\hline$<5$ cm & 18 & $8(44.4)$ & $10(55.6)$ & 0.591 \\
\hline$\geq 5$ cm & 60 & $31(51.7)$ & $29(48.3)$ & \\
\hline $\begin{array}{l}\text { Histologic } \\
\text { differentiation }\end{array}$ & & & & \\
\hline Well & 25 & $18(72.0)$ & $7(28.0)$ & 0.013 \\
\hline Moderate & 35 & $16(45.7)$ & $19(54.3)$ & \\
\hline Poor & 18 & $5(27.8)$ & $13(72.2)$ & \\
\hline Tumor depth & & & & \\
\hline T1/T2 & 14 & $5(35.7)$ & $9(64.3)$ & 0.238 \\
\hline T3/T4 & 64 & $34(53.1)$ & $30(46.9)$ & \\
\hline $\begin{array}{l}\text { Lymph node } \\
\text { metastasis }\end{array}$ & & & & \\
\hline N0 & 38 & $24(63.2)$ & $14(36.8)$ & 0.023 \\
\hline N1 & 40 & $15(37.5)$ & $25(62.5)$ & \\
\hline Stage & & $21(50.0)$ & $21(50.0)$ & 1.000 \\
\hline I/II & 42 & $18(50.0)$ & $18(50.0)$ & \\
\hline III & & & \\
\hline
\end{tabular}

*As determined by chi-square test

RNA junctions, and the lack of evidence of DNA-level rearrangements, together with the fact that these paired genes are adjacent genes in the genome, indicates that the chimera is most likely generated by read-through/splicing at RNA level.

Because the transcription of a chimeric RNA generated by read-through/splicing is controlled by the promoter of the 5' parental gene, the higher level of chimera in cancer raised the question if this is because that the 5' parental genes (GOLMI) is over-expressed in ESCC? To answer this question, we analyzed the available ESCC microarray gene expression database [17] in Oncomine. As shown in Figure 6B, the 5' parental gene GOLM1 were over-expressed in cancer tissue vs. matched benign (fold change $=3.4$ ) while the expression of 3 ' parental gene MAK10 remained unchanged. Therefore, the elevated chimera level in ESCC cancer tissue could at least in part be the consequence of over-expression of 5 ' parental gene and increased read-through transcription in cancer.

\section{DISCUSSION}

In this study, by employing multiple approaches we for the first time showed that GOLM1-MAK10, is highly enriched in ESCC. This chimera is likely generated through RNA processing and is unrelated to chromosomal rearrangements. Thus far, no recurrent fusion gene or chimeric RNAs have been identified in ESCC. Recurrent fusion genes have been described in many hematological cancers and solid tumors, and subsequently provided new insights into the molecular subtypes of cancers and pathogenesis. The biological consequences of chimeric RNAs however would be similar to that of gene fusions, as both mechanisms generate fusion transcripts that possess sequences from two parental genes. Transcription-induced chimeric RNAs thus, like fusion genes, could encode fusion proteins and contribute to oncogenesis. Our investigation demonstrated that chimeric RNAs are abundant in ESCC. In particular, GOLM1-MAK10 was recurrent among patients, and was enriched in tumor tissues at readily detectable levels, while near undetectable in normal esophageal tissue from subjects without esophageal neoplasia. This points to the important potential utility of GOLM1-MAK10 as molecular signatures for identifying ESCC and for early detection. In prostate cancer, the chimeric RNA SLC45A3ELK4 is present in urine at detectable levels $[7,8]$. This suggests that GOLM1-MAK10 could also be released by ESCC tumor into esophageal mucus or saliva protected by exosomes, thus serve as a non-invasive biomarker detectable using simple PCR-based assay.

Interestingly, our cell culture results demonstrated that chimera GOLM1-MAK10 identified in ESCC led to the translation of potentially functional fusion protein in human cells. GOLM1 (also known as GOLPH2 or GP73) is a Golgi membrane protein. The expression of GOLM1 gene is predominantly in epithelial cells [14], the cell type that ESCC is derived from. Previous reports indicated that elevated expression of parental GOLM1 is associated with localized prostate cancer [18] and HER$2 /$ neu proto-oncogene overexpression [19]. In addition, GOLM1 protein was found at high levels in the serum of patients with hepatocellular carcinoma [20, 21]. While GOLM1 was reported as a promising diagnostic cancer marker, its biological function is unclear [20,21]. Our observation revealed a close correlation between aberrant GOLM1-MAK10 and lymph node metastasis as well as tumor differentiation, implying a potential function in ESCC. The chimera GOLM1-MAK10 retains most of the 
GOLM1 sequence including the signal peptide region and the transmembrane domain that are required for targeting GOLM1 to the Golgi complex for secretion. This feature of secretion is particular important, considering that the most clinically useful cancer biomarkers to date, such as PSA for prostate cancer and CA125 for ovarian cancer, belong to the class of secreted proteins. Our findings that the aberrant chimera GOLM1-MAK10 was enriched in ESCC, and that GOLM1-MAK10 produced a secreted fusion protein, suggests the possibility of a unique protein signature detectable by standard non-invasive ELISA assays.

Intriguingly, approximately 18 of the 32 recurrent chimeric RNAs identified in prostate cancer were also present in ESCC (Figure 2), suggesting that similarity exists between the two cancer types. However, in ESCC the chimera GOLM1-MAK10 appears to be highly enriched while the two most prominent prostate cancer chimeric RNA, SLC45A3-ELK4 and TMEM79-SMG5 [6], and the fusion transcript from the prostate cancer fusion gene TMPRSS2-ERG [22], are undetectable in ESCC (Figure 2), indicating that the different cancer types posses different prominent molecular signatures with respect to chimeric RNA species.

GOLM1 is overexpressed in androgen-responsive Leydig cell tumor and prostate cancer [18, 23]. We performed bioinformatics analysis (http://alggen.lsi.upc. es/cgi-bin/promo_v3/promo/promoinit) and found that GOLM1 promoter region [14] spanned six androgenresponsive elements (AREs). It is known that hormones modulate fusion genes and their RNA products. For example, the expression of both chimeric TMPRSS2$E R G$ and SLC45A3-ELK4 are androgen-regulated in prostate cancer $[7,8]$, a male and androgen-sensitive cancer. Given that ESCC occurs predominantly in males and that sex steroid pathways such as androgen signaling may be involved in ESCC [24], we hypothesized that the expression of chimeric GOLM1-MAK10 may also be modulated by androgen. Our preliminary data suggest that androgen treatment of ESCC cell lines quickly elevated the expression of endogenous GOLM1-MAK10 within 12 hours, and maintained up to 48 hours, while the expression of GOLM1-MAK10 in untreated cells were unchanged during the same experimental period, indicating the regulation of the chimera expression is participated by androgen signaling (Supplementary Figure 1). The possibly hormone-dependent regulation of chimeric RNAs, once further elucidated, may better our understanding of molecular mechanisms responsible for ESCC carcinogenesis and potentially provides novel target pathways for therapeutic intervention.

In summary, this investigation shows that chimeric RNA, previously unknown in esophageal carcinoma, is prevalent in ESCC, and identified GOLM1-MAK10 as important molecular signature of ESCC. Chimeric RNAs may represent a novel class of molecular alteration in
ESCC that provides important insights into the molecular mechanism invovled in ESCC. Continued research of chimeric RNAs, in particular GOLM1-MAK10, will augment this and potentially provide novel target pathways for future therapies. The aberrant secreted fusion protein translated from GOLM1-MAK10 could serve as a unique protein signature detectable by standard noninvasive assays. This is critical, given that no clinically useful biomarker is currently available for screening this deadly disease or monitoring the treatment response.

\section{MATERIAL AND METHODS}

\section{Cell lines and human ESCC specimens}

The cell lines EC109, EC9706 (obtained from the Cell Bank of the Chinese Academy of Sciences, China), TE1 (kindly provided by Dr. X.C. Xu at UT M.D. Anderson Cancer Center, USA), and HKESC-2, and HKESC-2, HKESC-3, NE1 and NE3 cells (kindly provided by Dr. S.W. Tsao, University of Hong Kong) were prepared as described $[11,12]$. All human samples were obtained from cancer hospital of Shantou University Medical College in the Chaoshan littoral region located in southern China, which is recognized as one of the regions with the highest incidence of esophageal carcinomas in China. Study was approved by the institution ethics committee and specimens were collected with informed consent under an Institutional Review Board-approved protocol (IRB protocol number: \#04-070). A summary of clinicopathological information of patients is provided in Supplementary Table 1.

\section{RT-PCR, direct DNA sequencing and qRT-PCR}

Total RNA was extracted from tissues and cell lines using TRIzol reagent as per manufacturer's protocol (Invitrogen). RT-PCR was performed in human samples and cells as described previously [6]. The products of RT-PCR were gel purified and sequenced by Sanger sequencing to identify the exact fusion junctions of the candidate events [6]. qRT-PCR was performed using Absolute Blue QPCR SYBR Green low ROX mix (Thermo Scientific) on Applied Biosystems' 7500 realtime PCR system as described previously [6]. All assays were performed in triplicate and the results are shown as average fold change relative to GAPDH, which serves as an internal control. Primers used for qRT-PCR are listed in the online Supporting Information. 


\section{In situ hybridization}

Nonradioactive in situ hybridization was performed as described previously [13]. Briefly, antisense RNA probes labeled with digoxigenin were transcribed from cDNA templates. Paraffin-fixed tissue sections from ESCC were deparaffinized, treated with proteinase K, prehybridized and then hybridized with probes. Following hybridization, the slides were washed and treated with a blocking reagent and then incubated with anti-digoxigenin and detected as detailed [13]. Antisense probes were designed against the fusion junction or RNA segments specific to these chimeric RNAs, so as to distinguish chimeric RNAs from the parental mRNAs. We tested and confirmed the specificity of the antisense probes to distinguish chimeric RNAs from parental mRNA using dot blot assay. Antisense probes used for in situ hybridization are listed in online Supporting Information.

\section{Long-range PCR}

LA PCR kit (Takara) was used for long-range PCR analysis of patient genomic DNA as described [6]. To ensure that the PCR primers designed can amplify the intended DNA fragment, we used commercial human genomic DNA (Promega) as a control. Primers used are listed in the online Supporting Information.

\section{Cloning and transfection of chimera}

Using the primers (listed in the online Supporting Information), RT-PCR was performed on patient samples to generate the full length GOLM1-MAK10. A FLAG tag was then added to the $\mathrm{C}$-terminus of this PCR product and cloned into the vector HDM. HEK-293T cells were transfected with this plasmid using TransIT- 293 reagent (Mirus) according to the manufacturer's protocol.

\section{Western blotting}

48 hours after transfection of cloned full-length GOLM1-MAK10, proteins within transfected cells were extracted using RIPA buffer (Santa Cruz Biotechnology). Western blotting was performed using the following antibodies: Anti-FLAG (SIGMA Catalog number F1804) and Anti- $\beta$-actin (SIGMA Catalog number A2228).

\section{ACKNOWLEDGEMENTS}

We thank Stephanie Pangas and Matthew H. Wilson for critical suggestions. Hao Zhang is supported in part by the National Natural Science Foundation of China (81071736 and 30973508) and the Research Fund for the Doctoral Program of Higher Education of China (RFDP,
20104402110005). Laising Yen is supported by DOD W81XWH-10-10327, OCRF PPD/BCM/01.12, NIH R01EB013584. Kalpana Kannan is supported by PCF Young Investigator award.

\section{Author contributions:}

W. L., L. L., J. L., P-W. C., Y. W. performed experiments; K. K. cloned the chimeric transcript and performed fusion protein assay; W. L., K. K., L. L., L. Y. and H. Z. analyzed data; Y-P. C. and J. G. contributed reagents; L. Y. and H. Z. designed research and wrote the paper.

\section{REFERENCES}

1. Parkin DM, Bray F, Ferlay J and Pisani P. Global cancer statistics, 2002. CA Cancer J Clin. 2005; 55(2): 74-108.

2. Parkin DM, Bray F, Ferlay J and Pisani P. Estimating the world cancer burden: Globocan 2000. Int J Cancer. 2001; 94:153-56.

3. Enzinger PC and Mayer RJ. Esophageal cancer. N Engl J Med. 2003; 349(23):2241-52.

4. Gingeras TR. Implications of chimaeric non-co-linear transcripts. Nature. 2009; 461(7261):206-11.

5. Kaye FJ. Mutation-associated fusion cancer genes in solid tumors. Mol Cancer Ther. 2009; 8(6):1399-408.

6. Kannan K, Wang L, Wang J, Ittmann MM, Li W and Yen L. Recurrent chimeric RNAs enriched in human prostate cancer identified by deep sequencing. Proc Natl Acad Sci U S A. 2011; 108(22):9172-77.

7. Maher CA, Kumar-Sinha C, Cao X, Kalyana-Sundaram S, Han B, Jing X, Sam L, Barrette T, Palanisamy N and Chinnaiyan AM. Transcriptome sequencing to detect gene fusions in cancer. Nature. 2009; 458(7234):97-101.

8. Rickman DS PD, Moss B, VanDoren VE, Chen CX, de la Taille A, Kuefer R, Tewari AK, Setlur SR, Demichelis F and Rubin MA. SLC45A3-ELK4 is a novel and frequent erythroblast transformation-specific fusion transcript in prostate cancer. Cancer Res. 2009; 69:2734-38.

9. Frenkel-Morgenstern M, Lacroix V, Ezkurdia I, Levin Y, Gabashvili A, Prilusky J, Del Pozo A, Tress M, Johnson R, Guigo R and Valencia A. Chimeras taking shape: potential functions of proteins encoded by chimeric RNA transcripts. Genome research. 2012; 22(7):1231-42.

10. Druker BJ, Guilhot F, O’Brien SG, Gathmann I, Kantarjian H, Gattermann N, Deininger MW, Silver RT, Goldman JM, Stone RM, Cervantes F, Hochhaus A, Powell BL, Gabrilove JL, Rousselot P, Reiffers J, et al. Five-year follow-up of patients receiving imatinib for chronic myeloid leukemia. N Engl J Med. 2006; 355(23):2408-17.

11. Cheung PY, Deng W, Man C, Tse WW, Srivastava G, Law S, Tsao SW and Cheung AL. Genetic alterations in a telomerase-immortalized human esophageal epithelial cell 
line: implications for carcinogenesis. Cancer Lett. 2010; 293(1):41-51.

12. Hu YC, Lam KY, Law SY, Wan TS, Ma ES, Kwong YL, Chan LC, Wong J and Srivastava G. Establishment, characterization, karyotyping, and comparative genomic hybridization analysis of HKESC-2 and HKESC-3: two newly established human esophageal squamous cell carcinoma cell lines. Cancer genetics and cytogenetics. 2002; 135(2):120-27.

13. Zhang H, Singh RR, Talukder AH and Kumar R. Metastatic tumor antigen 3 is a direct corepressor of the Wnt4 pathway. Gene Dev. 2006; 20(21):2943-48.

14. Gong Y, Long Q, Xie H, Zhang T and Peng T. Cloning and characterization of human Golgi phosphoprotein 2 gene (GOLPH2/GP73/GOLM1) promoter. Biochem Biophys Res Commun. 2012; 421(4):713-20.

15. Nonn L, Ananthanarayanan V and Gann PH. Evidence for field cancerization of the prostate. The Prostate. 2009; 69(13):1470-79.

16. Kim HJ, Lv D, Zhang $\mathrm{Y}$, Peng $\mathrm{T}$ and Ma X. Golgi phosphoprotein 2 in physiology and in diseases. Cell \& bioscience. 2012; 2(1):31.

17. Hu N, Clifford RJ, Yang HH, Wang C, Goldstein AM, Ding T, Taylor PR and Lee MP. Genome wide analysis of DNA copy number neutral loss of heterozygosity $(\mathrm{CNNLOH})$ and its relation to gene expression in esophageal squamous cell carcinoma. BMC genomics. 2010; 11:576.

18. Varambally S, Laxman B, Mehra R, Cao Q, Dhanasekaran S, Tomlins SA, Granger J, Vellaichamy A, Sreekumar A, Yu JJ, Gu WJ, Shen RL, Ghosh D, Wright LM, Kladney RD, Kuefer R, et al. Golgi Protein GOLM1 Is a Tissue and Urine Biomarker of Prostate Cancer. Neoplasia. 2008; 10(11):1285-U1104.

19. Oh JJ, Grosshans DR, Wong SG and Slamon DJ. Identification of differentially expressed genes associated with HER-2/neu overexpression in human breast cancer cells. Nucleic acids research. 1999; 27(20):4008-17.

20. Marrero JA, Romano PR, Nikolaeva O, Steel L, Mehta A, Fimmel CJ, Comunale MA, D'Amelio A, Lok AS and Block TM. GP73, a resident Golgi glycoprotein, is a novel serum marker for hepatocellular carcinoma. J Hepatol. 2005; 43(6):1007-12.

21. Mao YL, Yang HY, Xu HF, Lu X, Sang XT, Du SD, Zhao HT, Chen W, Xu Y, Chi TY, Yang ZY, Cai JQ, Li H, Chen JG, Zhong SX, Mohanti SR, et al. Golgi protein 73 (GOLPH2) is a valuable serum marker for hepatocellular carcinoma. Gut. 2010; 59(12):1687-93.

22. Tomlins SA, Rhodes DR, Perner S, Dhanasekaran SM, Mehra R, Sun XW, Varambally S, Cao X, Tchinda J, Kuefer R, Lee C, Montie JE, Shah RB, Pienta KJ, Rubin MA and Chinnaiyan AM. Recurrent fusion of TMPRSS2 and ETS transcription factor genes in prostate cancer. Science. 2005; 310(5748):644-48.

23. Fritzsche FR, Kristiansen G, Riener MO, Dietel M and
Oelrich B. GOLPH2 expression may serve as diagnostic marker in seminomas. Bmc Urol. 2010;10.

24. Tihan T, Harmon JW, Wan X, Younes Z, Nass P, Duncan KL and Duncan MD. Evidence of androgen receptor expression in squamous and adenocarcinoma of the esophagus. Anticancer research. 2001; 21(4B):3107-14. 